\title{
Tuberculosis en pacientes ancianos
}

“...era moda sufrir de los pulmones,, todo el mundo pade cía la enfermedad, principalmente los poetas..."

\section{Memorias de Alejandro Dumas}

Desde que Chopín, Dostoiewski, Keats, Allan Poe, Voltaire y otros muchos, padecieron y murieron de tuberculosis, la dolencia fue tema de interés en el campo de las Letras, destacando Thomas Mann con La Montaña Mágica, Cela con Pabellón de Reposo, y más recientemente Manuel Rivas, en $O$ Lapis do Carpinteiro retoma el viejo tema de la belleza tísica. A causa de la evolución de las ciencias, la enfermedad dejó de pertenecer a almas románticas y de artistas -quienes crearon el eufemismo belleza tísica - y pasó a ser una enfermedad de los cuerpos, de la baja esfera social, que a comienzos de la era industrial, vivían hacinados y trabajaban en paupérrimas condiciones.

Al hallazgo del germen causal comunicado por Koch en Berlín el 24 de marzo de 1882, siguieron múltiples estudios y descubrimientos. Pero fue a partir de los años 1944 (Waskman descubre la estreptomicina y Lehmann el ácido ParaAminoSalcílico: PAS), 1950 (se descubre la isoniacida) y 1953 (se inicia la quimioterapia de larga duración), cuando comienza el declinar masivo de la mortalidad y de nuevos casos (1).

Entre las características de la enfermedad tuberculosa figuran su período de incubación muy variable, sus manifestaciones clínicas proteiformes (2-4) y su duración y persistencia $(1,5,6)$. Una vez que penetra en el organismo puede permanecer silente de por vida o desarrollar enfermedad cuando la situación inmunológica lo permita, como sucedió siempre en la senectud (7-10) y modernamente con el SIDA $(4,7,9,11$ 20).

Dado que la tuberculosis sigue siendo hoy un problema sociosanitario notable en nuestro medio, dado que el contingente geriátrico representa una parte considerable de la población, doblemente expuesta a padecer la enfermedad, bien por la reactivación de una forma antigua, bien por enfermedad exógena, y dado que sus formas de presentación parecen diferir de las habituales, diversos autores siguen investigando en torno a la tuberculosis en pacientes ancianos.
Seguramente, en un intento de aclarar diversas cuestiones clínicas, aparece el interesante y oportuno trabajo suscrito por Lado y cols., (21). Con su numerosa casuística, los autores nos dan a conocer la distribución y formas de presentación de la enfermedad tuberculosa en una población mayor de 65 años en un área endémica. En este trabajo creemos necesario destacar: a) el no haberse registrado ningún caso de asociación con infección por VIH; b) la baja frecuencia de cavitación en las formas pulmonares; c) la superación de las expectativas esperadas en cuanto a las formas extrapulmonares y d) el cambio significativo en la presentación habitual de la enfermedad.

Diversos autores han encontrado diferencias significativas en tuberculosis pulmonar (22-26) y pleural (26) entre poblaciones ancianas y jóvenes. Pese a que en un estudio con meta-análisis (27) se concluyó que las principales diferencias encontradas -también en tuberculosis pulmonar- en los pacientes ancianos en relación con los más jóvenes, se pueden explicar a través de cambios fisiopatológicos conocidos que acontecen con la edad, algunos autores han sugerido que, la tuberculosis pulmonar del anciano, es una entidad diferente (28-30) e importante de identificar, tanto desde los puntos de vista inmunológicos, como epidemiológicos, clínicos y del tratamiento.

\section{INMUNOLOGÍA Y FISIOPATOLOGÍA}

Con la edad se produce un declive en la protección inmunológica tanto en la producción de anticuerpos de alta afinidad, como disminución de la memoria inmune en respuesta a la vacunación y de hipersensibilidad retardada. En la senescencia, además de la respuesta de las células T, que contribuyen a la defensa contra las infecciones con la producción de patrones específicos de citoquinas, hay que tener en cuenta otros factores extrínsecos frecuentes y negativos asociados a la edad y a la tuberculosis, tales como dieta inapropiada, mal estado de nutrición, escasa actividad física y co-morbilidad (7,8-10,31-37). Se han descrito infecciones parasitarias asintomáticas que asociadas a tuberculosis y otros estados de inmunosupresión, pueden conducir a una respuesta de activación genética de citoquinas tipo 2 (36), en vez de la respuesta de citoquinas tipo 1 , que sería la adecuada contra la tuberculo- 
sis, y podría ser una explicación de casos con extensa enfermedad tuberculosa (37). De la misma manera, una disminución en los niveles de citoquinas previamente elevados puede ser indicadora de buena respuesta al tratamiento (32).

Se acepta que el proceso de envejecimiento se puede manifestar en varias áreas inmunológicas:

1. En los centros germinales, parece ser que hay disminución del procesamiento de complejos inmunes, disminución de tráfico de células dendríticas $(10,34)$ y disminución de hipermutaciones de inmunoglobulinas (10).

2. En el cortex, afecta a la producción de linfocitos $\mathrm{T}$. Aunque en sangre periférica no se modifican, hay pérdidas cualitativas con linfocitos $\mathrm{T}$ en estadío postmaduro, sin capacidad de replicación e incapaces de realizar su función inmunocompetente de manera adecuada, con menor actividad de los CD4+ cooperadores, probable mayor actividad de los fenotipos CD8+ supresores y menor actividad de CD8 citotóxicos. En cuanto a la producción de IL-4 (Interleuquina 4), de IFN- $\gamma$ (Interferon $\gamma$ ) y a la relación CD4/CD8, hay discrepancias, pues mientras algunos autores las han encontrado disminuidas, otros aumentadas (10). También se ha descrito aumento de las células $\mathrm{T}$ de memoria, descenso de células "inexpertas", disminución de las señales de calcio, de su movilización y de la fosforilización proteíca (10).

3. En los folículos primarios, donde se describe falta de hipermaduración de los linfocitos $\mathrm{T}$, quizá por una diminución en la expresión de la molécula B7-2, a través de la cual las células B reciben las señales de activación y diferenciación, de la molécula de superficie CD28 de las células T. Estos defectos en ambas células, $\mathrm{T}$ y $\mathrm{B}$, parecen contribuir al declive en la hipermutación de anticuerpos y descenso en la inmunidad humoral. También, con la edad, se describe una pérdida en la habilidad para producir anticuerpos ante nuevos antígenos, con aumento de autoanticuerpos y de inmunocomplejos circulantes y pérdida de la capacidad de duplicación ante la estimulación con mitógenos, por menor capacidad de fosforilización (10).

En el caso de la tuberculosis, los bacilos llegados a los alveolos, en las "gotitas de Flügge", son fagocitados por los macrófagos alveolares. Si la población bacilar es escasa y su virulencia baja pueden ser destruidos y la infección controlada, pero si hay muchos bacilos o son muy virulentos pueden sobrevivir dentro del macrófago e incluso destruirlo, liberándose los enzimas macrofágicos y las proteínas bacilares, dando lugar a una respuesta inflamatoria inespecífica, con aumento de la permeabilidad capilar y reclutamiento de linfocitos $\mathrm{T}$, con exudado alveolar y migración desde la sangre de neutrófilos, linfocitos y mononucleares.

Algunas células cargadas de bacilos pueden escapar del foco alveolar y llegar a los ganglios regionales, donde tiene lugar la interacción entre los antígenos bacilares y el sistema inmunitario. Desde aquí, aún otro contingente de bacilos puede pasar a la sangre -bacteriemia primaria-, y van a parar a los más insospechados lugares de la economía, entre ellos el intersticio pulmonar: tuberculosis miliar de diversa evolución.

En el sistema inmunitario, los macrófagos, al mostrar los antígenos dan lugar a una proliferación clonal de linfocitos $\mathrm{T}$ que se diferencian en: linfocitos $\mathrm{T}$ de memoria, linfocitos $\mathrm{T}$ cooperadores $(\mathrm{CD} 4+)$ y linfocitos $\mathrm{T}(\mathrm{CD}+8)$ citotóxicos $\mathrm{y}$ supresores.

Cuando los linfocitos T CD8+ supresores muestran gran actividad con poca respuesta de los CD4+, como puede ocu- rrir con la edad, se liberaran grandes cantidades de bacilos y aparecen formas graves de la enfermedad.

Los linfocitos $\mathrm{T}$ de memoria poseen la función de vigilancia inmunológica y su persistencia hace posibles posteriores respuestas rápidas y adecuadas ante una nueva entrada bacilar (3). Este proceso, que ocurre entre 2 y 10 semanas de la entrada del bacilo, marcará la positividad en la prueba de la tuberculina.

Otras células pueden participar en la contención bacteriana, tales como células dendríticas, en gran cantidad en las vías aéreas, y que disminuyen con la edad por derivarse hacia la formación de monocitos, que aún son capaces de poner en marcha una función residual en las células T senescentes (34). Con todo, el mayor papel parece ser el que desempeñan los monocitos y macrófagos. Los Th1 (linfocitos T tipo 1 CD4+ $\alpha \beta$ y linfocitos natural killer T NK), también conocidos como productores de linfocinas o citoquinas tipo 1 , como IFN- $\gamma$, y factor de necrosis tumoral (TNF- $\alpha$ ) y otros, activan a los macrófagos alveolares. Así los fagosomas, que han englobado bacterias, las aniquilan por varios mecanismos, tales como la fusión fagosoma-lisosoma o la producción de sustancias reactivas al oxígeno y al nitrógeno, particularmente óxido nítrico, como ocurre con la Nramp (natural resistance associated macrophage protein), que transporta nitrito desde un compartimento intracelular menos ácido, como el citosol, a otro más ácido, como un fagosoma, donde es convertido en NO (8). También se han descrito macrófagos alveolares humanos unos con óxido nítrico sintectasa y otros con alta capacidad para generar oxígeno reactivo, particularmente $\mathrm{H}_{2} \mathrm{O}_{2}$ (8). Estos mecanismos darían lugar a inhibición del crecimiento bacteriano y/o eliminación de mycobacterias tras la apoptosis o muerte celular programada. Esta defensa, por parte de los macrófagos, los NK y los neutrófilos alcanzaría el control de la infección antes de desarrollar la respuesta inmune adquirida (3).

Más adelante, tras la confrontación en el sistema inmune, la mayoría de individuos pasarán a la situación de infección clínicamente latente, describiéndose como buena respuesta la que se acompaña de elevación de valores plasmáticos de IFN$\gamma$ (32). Éste y otros factores también parecen jugar un importante papel en la tuberculosis pleural, con valores, en el líquido pleural del IFN- $\gamma$ y del porcentaje de células que lo producen, mucho más elevados que en la sangre $(3,38)$.

Pero la tuberculosis se puede activar en el transcurso de la vida, especialmente como consecuencia de las alteraciones en la respuesta inmune de la vejez. Hay estudios que demuestran que la proliferación bacteriana podría ser debida a la capacidad de las micobacterias para producir catalasas y amoníaco, que alcalinizaría el medio intralisosomal y se inhibiría la fusión fagosoma-lisosoma.

Los macrófagos también segregan otras citoquinas como la IL-2, la IL-12 y la IL-18, cuyos niveles se pueden medir en plasma y se describen más elevados en la tuberculosis que en otras condiciones inflamatorias (32), o las citoquinas tipo 2: la IL-4, la IL-5, que inhiben la función macrofágica, aunque no hay evidencia de que éstas abunden en los pulmones de pacientes con tuberculosis, quizá por efecto supresivo de los Th2 (linfocitos T tipo 2 CD4+). En otras palabras, los linfocitos $\mathrm{T}$ cooperadores pueden diferenciarse en dos fenotipos, según el tipo de citoquinas segregadas en Th1, que segregan IFN- $\gamma$, IL-2 e IL-18, que activan macrófagos y monocitos, y Th2 que producen IL-4 e IL-5, con reclutamiento de eosinófilos y producción de IgE. Ambas son reac- 
ciones inflamatorias, pero mientras la reacción Th1 representa una respuesta de inmunidad celular retardada, protectora en el caso de la tuberculosis, igual que la que ocurre con el rechace de un trasplante, la reacción Th2 representa una respuesta inmunitaria humoral, con escasa formación de granulomas, menos adecuada ante la infección tuberculosa, con anergia cutánea, títulos altos de anticuerpos y elevada población bacilar, que se asociaría con tuberculosis miliar, meningitis y otras formas graves de la enfermedad, de relativa alta frecuencia en la población de mayor edad. El paradigma de este mecanismo lo representa la lepra lepromatosa (2-4,710,31-37)

En la respuesta inmune contra la tuberculosis las células T CD8+, parece que están involucradas en la lisis y apoptosis, y también serían capaces de segregar citoquinas, tales como IFN- $\gamma$ y IL-4 $(8,10)$.

Finalmente, el papel de otras subclases de células T como las $\mathrm{T} \gamma / \delta$, en la respuesta del huesped, sigue siendo tema de estudio $(8,10)$, aunque funcionalmente parece que se comportan de manera similar a las CD4+ (3).

Otro hecho destacado en la respuesta a las micobacterias es la formación de granulomas como respuesta a los antígenos de la pared bacteriana y adyuvantes. Experimentalmente se ha visto que citoquinas quimiotácticas originan una migración de neutrófilos hacia el lugar de inoculación, seguida de monocitos, que se diferencian en macrófagos a los 2-3 días, con formación de grandes granulomas de macrófagos maduros y células epitelioides a los 5-7 días y células gigantes de Langerhan hacia el $9^{\circ}$ día. Ya en granulomas plenamente desarrollados, entre las células epitelioides, en apoptosis, se observan células dendríticas con filopodia, y en la periferia células T CD4+ y CD8+. In situ se describe proliferación bacteriana tanto en los linfocitos como en los macrófagos, con adhesión celular homotípica y heterotípica, mediada por la molécula de adhesión intercelular ICAM-1 (molécula de superficie regulada por M. tuberculosis) y que puede se amplificada por citoquinas, tales como TNF- $\alpha$, IL6 e IFN- $\gamma(3,8)$.

\section{EPIDEMIOLOGÍA Y CLÍNICA}

Como se ha comunicado en diversos estudios, en nuestro medio, la incidencia de la enfermedad es alta, con dos ondas de mayor incidencia, una entre los 15 y los 35 años y otra después de los 65 años (13-15,19).

Los centros cerrados, especialmente geriátricos, suponen un incremento en la transmisión bacilar. El estudio del genoti- po del DNA bacteriano nos puede permitir identificar subtipos moleculares del bacilo y genes mutantes con resistencias, como en los casos recientemente publicados de multiresistencias (2-4).

Desde el punto de vista clínico, si la tuberculosis se ha definido como la gran simuladora, esto es particularmente cierto en pacientes inmunodeprimidos y en mayores de 65 años. En esta población, con enfermedad pulmonar, se han descrito diferentes formas de presentación radiológica que en los menores de 65 años, pero lo más destacado es la presentación de enfermedad de localización extrapulmonar, así como formas infrecuentes (39), crípticas o diseminadas (40). Todo ello, junto con la alta comorbilidad de esta población puede conducir a retrasos diagnósticos con elevada morbi-mortalidad, no siendo infrecuente que el diagnóstico final nos venga dado por el informe de necropsia $(41,42)$.

\section{TRATAMIENTO}

El tratamiento de la tuberculosis del anciano no difiere del de la población adulta, si bien hay que tener en cuenta que con la edad aumentan los casos de efectos adversos, aunque en estudios comparativos no se han encontraron diferencias significativas en relación con la población adulta (43). En el improbable caso de aparición de resistencias se están estudiando los nuevos macrólidos, quinolonas, interferón gamma y subclases de linfocitos T CD4+ (44).

Tanto en el tratamiento curativo como en el preventivo la hepatitis por isoniacida aumenta con la edad, sin diferencias entre hombres y mujeres, sin embargo, a pesar de este y otros efectos adversos está claramente demostrado el beneficio versus perjuicio del tratamiento (45).

Al igual que Lado y cols. (21), concluimos resaltando la impresión de que se está produciendo un cambio en la forma de presentación de la enfermedad tuberculosa en pacientes ancianos y enfatizamos sobre la necesidad de obtener un diagnóstico precoz, pues como vimos, por motivos inmunológicos, epidemiológicos y clínicos este grupo de población es especialmente vulnerable.

M. SALGUEIRO RODRíGUEZ Servicio de Neumología Hospital Clínico Universitario. Santiago de Compostela

\section{Bibliografía}

1. Pereira Poza A. La paciencia al sol. Ediciós Do Castro, 1999. Sada A Coruña.

2. Iseman MD, Huitt GA. Tuberculosis. Clin Chest Med 1997; 18.

3. Hershfield ES. Tuberculosis. Edited by Reichman LB, 2nd Edition, 2000, Marcel Dekker, Inc. New York. Basel.

4. American Thoracic Society. Diagnostic Standars and Classification of Tuberculosis in Adults and Children. Am J Respir Crit Care Med, 2000; 161: 1376-95.
5. Actas de la Reunión "Tuberculose. Un século". Conmemoraçao do 90 Aniversario do $2^{\circ}$ Congresso Contra a Tuberculose. Viana do Castelo (Portugal), 1992.

6. Grande Enciclopedia Portuguesa e Brasileira, 1967; XXXIII: 114. Lisboa, Río de Janeiro.

7. Gómez de la Concha E. II: Envejecimiento del sistema inmunológico. En Ribera Casado JM, Gil Gregorio P. VIII Patología Infecciosa en Geriatría. Clínicas Geriátricas 1992; 108-117. Editores Médicos S.A., Madrid. 
8. Schluger NW, Rom WN. State of the Art. The host immune response to tuberculosis. Am J Respir Crit Care Med 1998; 157: 679-91.

9. Sherman S. Human aging et the Millennium. En: Rosen CJ, Glowacki J and Bilezikian JP. The Aging Skeleton 1999; 11-20. Academic Press, San Diego USA.

10. Miller RA. The aging immune system: primer and prospectus, Science 1997; 273: 70-4.

11. Caso Pita MC. XI: Tuberculosis en el anciano. En Ribera Casado JM, Gil Gregorio P. VIII Patología Infecciosa en Geriatría. Clínicas Geriátricas, Editores Médicos S.A., Madrid 1992; 108-117.

12. Brudney K, Dobkin J. Resurgent tuberculosis in New York City. Human immunodeficiency virus, homelessness, and the decline of tuberculosis control programs. Am Rev Respir Dis 1991; 144: 745-9.

13. Salgueiro Rodríguez M, Zamarrón Sanz C, Álvarez-Calderón Prat P, et al. Estudio epidemiológico de la tuberculosis en el área sanitaria de Santiago de Compostela durante los años 1989, 1990 y 1991. An Med Interna (Madrid) 1993; 10: 427-32.

14. Salgueiro Rodríguez M, Zamarrón Sanz C, Otero Y, et al. Estudio epidemiológico de la tuberculosis en el área sanitaria de Santiago de Compostela durante los años 1992, 1993 y 1994. An Med Interna (Madrid) 1996; 13: 111-4

15. Salgueiro Rodríguez M, Zamarrón Sanz C, González Barcala J, et al. Estudio epidemiológico de la tuberculosis en el área sanitaria de Santiago de Compostela durante los años 1995, 1996, 1997 y 1998. An Med Interna (Madrid) 2001; 18: 20-3.

16. Martínez Vázquez JM, Cabarcos Ortíz de Barrón A, Barrio Gómez E. Tuberculosis e infección por VIH: epidemiología (primera de tres partes) An Med Interna (Madrid) 1997; 14: 253-6.

17. Martínez Vázquez JM, Cabarcos Ortíz de Barrón A, Barrio Gómez E. Tuberculosis e infección por VIH: patogenia (segunda de tres partes) An Med Interna (Madrid) 1997; 14:310-6.

18. Martínez Vázquez JM, Cabarcos Ortíz de Barrón A, Barrio Gómez E. Tuberculosis e infección por VIH: de la clínica a la prevención (tercera de tres partes) An Med Interna (Madrid) 1997; 14: 363-8.

19. Anibarro García R, Vázquez-Gallardo R, Toubes Navarro ME, et al. Epidemiología de la tuberculosis en Galicia. An Med Interna (Madrid) 1999; 16: 290-6.

20. Lado Lado FL, Barrio Gómez E, Carballo Arceo E, Cabarcos Ortíz de Barrón A. Tuberculosis e infección por el virus de la inmunodeficiencia humana: manifestaciones clínicas y rendimiento de procedimientos diagnósticos según las distintas formas de localización de la enfermedad. An Med Interna (Madrid) 2000; 17: 27-34

21. Lado Lado FL, Túñez Bastida V, Golpe Gómez AL, Cabarcos Ortíz de Barrón A, Pérez del Molino ML. Tuberculosis en pacientes ancianos. Formas de presentación. An Med Interna (Madrid) 2002; 19: 111-4.

22. Morris CD. Sputum examination in the screening and diagnosis of pulmonary tuberculosis in the elderly. Q J Med 1991; 81: 999-1004.

23. Umeki S. Comparison of younger and elderly patients with pulmonary tuberculosis. Respiration 1989; 55: 75-83.

24. Zamarrón C, Salgueiro M, Álvarez JM, Otero Y, Rodríguez Suárez. Características clínicas de la tuberculosis pulmonar en el anciano. An Med Interna (Madrid) 1997; 14: 167-9.

25. Katz PR, Reichman W, Dube D, Feather J. Clinical features of pulmonary tuberculosis in young and veterans. J Am Geriatr Soc 1987; 35: 512-5.

26. Korzeniewska-Kosela M, Krysl J, Muller N, Black W, Allen E, FitzGerald JM. Tuberculosis in young and the elderly. A prospective comparison study. Chest 1994; 106: 28-32.
27. Pérez-Guzmán C, Vargas MH, Torres-Cruz A, Villarreal-Velarde H. Does aging modify pulmonary tuberculosis? A meta-analytical reviw. Chest 1999; 116: 961-7.

28. Stead WW. Tuberculosis among elderly persons: an outbreak in a nursing home. Ann Inter Med 1981; 94: 606-10.

29. Morris CD. The radiography, haematology and biochemistry of pulmonary tuberculosis in aged. Q J Med 1989; 266: 529-35.

30. Morris CD. Pulmonary tuberculosis in the elderly. Thorax 1990; 45: 912-3.

31. Juffermans NP, Verbon A, Van Deventer SJH, Deutekom H, Speelman $\mathrm{P}$, Van der Poll T. Tumor Necrosis Factor and Interleukin-1 Inhibitors as markers of disease activity of tuberculosis. Am J Respir Crit Care Med 1998; 157: 1328-31.

32. Barnes PF, Wizel B. Editorial. Tipe 1 Cytokines and pathogenesis of tuberculosis. Am J Respir Crit Care Med 2000; 161: 1773-4.

33. Tsao TC, Hong J, Li LF, Hsich MJ, Liao SK, Chang KS. Imbalances between Tumor Necrosis Factor-alpha and its soluble receptor forms, and Interleukin-1 beta and Interleukin-1 Receptor Antagonist in BAL fluid cavitary pulmonary tuberculosis. Chest 2000; 117: 103-9.

34. Lung TL, Saurwein-Teiss1 M, Paarson W, Schonitzer D, Grubeck_Loebenstein B. Unimpared dendritic cells can be derived from monocytes in old age and can mobilize residual function in senescent $\mathrm{T}$ cells. Vaccine 2000; 18: 1606-16112.

35. Yamada G, Shijubo N, Shigehara K, Okamura H, Kurimoto M, Abe S. Increased levels of circulating interleukin 18 in patients with avanced tuberculosis. Am J Respir Crit Care Med 2000; 161: 1786-9.

36. Bentwich Z, Kalinkovich A, Weisman Z, Borkow G, Beyers N, Beyers AD. Can erradication of hemiltic infections change the face of AIDS and tuberculosis? Immunol Today 1999; 20: 485-7.

37. Seah GT, Scott GM, Rook GAW. Type 2 cytokine gene activation and its relationship to extent of disease in patients with tuberculosis. J Infect Dis 2000; 181: 385-9.

38. Valdés L, San José E, Álvarez D, et al. Diagnosis of tuberculosis pleurisy using the biologic parameters Adenosine Deaminase, Lysozyme and Interferon Gamma. Thorax 1993; 103: 458-65.

39. Pène F, Papo T, Brdy-Gulphe L, Cariou A, Piette JC, Vinsonneau C. Seeptic Shock and Thrombotic microangiopathy due to Mycobacterum tuberculosis in a nonimmunocompromised patient. Arch Intern Med 2001; 161: 1347-8.

40. Lado Lado FL, Túñez Bastida, Golpe Gómez A, Ferreiro Regueiro JM, Cabarcos Ortíz de Barrón A. Tuberculosis extrapulmonar. Formas de presentación en nuestro medio. An Med Interna (Madrid) 2000; 17: 637-41.

41. Conthe P, Gaspar G, et al. El problema de la tuberculosis miliar: estudio de 20 casos no diagnosticados hasta la autopsia. Med Clin (Barc) 1982; 79: 268-72.

42. Veiga González M, Riestra Martínez, et al. Tuberculosis miliar, Estudio autópsico de 29 casos. An Med Interna (Madrid) 1995; 12: 17-20.

43. Aguilar X, Ruiz J, Frenández-Muixi J, Teixido A, et al. Eficacia y Tolerancia del tratamiento de tuberculosis en ancianos. Arch Bronconeumol 1996; 32: 122-6.

44. Collazos J, Martínez E, Mayo J, Riñón M. Response of lynfocyte subsets in patients under treatmen for tuberculosis. Eur J Clin Microbiol Infect Dis 2000; 19: 623-6.

45. Stead WW, To T, Harrison R, Abraham JH. Benefit-risk considerations in preventive treatment for tuberculosis in elderly persons. Ann Intern Med 1987; 107: 843-5. 